\title{
A method for using one finger to press three separate keys on a three-dimensional keyboard designed to be mounted on a mouse
}

\author{
Tomohiro Suzuki ${ }^{*}$, Satoshi Miura' ${ }^{1}$, Yo Kobayashi ${ }^{2}$ and Masakatsu G. Fujie ${ }^{2}$
}

\begin{abstract}
Here we propose and verify the feasibility of a new keyboard structure for keyboard-mounted mouse. Reducing the distance that fingers move while typing within a three-dimensional (3-D) key arrangement that fits along the fingers is effective for keyboard-mounted mouse, provided that each finger can separately press three different keys. Our objective was to design and test a method for separately being able to press three keys with a single finger, and in the process, reduce finger-moving distance. We analyzed 3-D finger motion while participants typed on a standard keyboard, and used this data to develop a 3-D layout for keys that are arranged along the fingers. Analysis of 3-D finger motion while using our new keyboard showed that the distance fingers traveled was $74 \%$ less than that when using a standard keyboard $(p<0.05)$. Moreover, this did not result in typing mistakes caused by interference between the keys and finger movements. After typing 30 characters 20 times, the average input error rate in the 20th trial was $18 \%$, while the average error rate across all trials for the standard keyboard was $9.5 \%$. We conclude that our proposed 3-D keyboard can be used accurately with one finger while reducing the distance fingers must move. However, input mistakes were caused by finger linkage motion. In the future, we must devise a character-input algorithm that eliminates such erroneous input. We must also include the mouse function and evaluate the operability of the device in tasks that require keyboard and mouse use.
\end{abstract}

Keywords: Keyboards, Motion analysis, User interfaces, Human computer interaction, Ergonomics

\section{Background}

\section{Social background}

In the current information-technology society, people spend a great deal of time using computers at work and at home. Keyboards and mice are widely used as the de facto standard for operating personal computers, and almost all people are accustomed to using them [1]. Generally, keyboards are used for character input and mice are used to direct the cursor and make selections. However, because hands must be frequently moved between the two independent interfaces, typing efficiency and mouse operation are not as efficient as they could be. Thus, integrating the two interfaces, mounting

\footnotetext{
*Correspondence: suzuki-t03@toki.waseda.jp

${ }^{1}$ The Graduate School of Science and Engineering, Waseda University,

Tokyo, Japan

Full list of author information is available at the end of the article
}

mouse functions on the keyboard or mounting keyboard functions on the mouse, will be effective to reduce the movement between two interfaces and will improve the efficiency of typing and mouse operation. Developing an interface that combines keyboard and mouse function is therefore of keen interest.

\section{Related works}

TrackPoint is one of several interfaces that have integrated devices that mount mouse functions on the keyboard. The TrackPoint has a mouse trackpad that is placed in the center of the keyboard [2]. By using the TrackPoint, mouse-cursor movement can be controlled by using the fingertips. Another keyboard can sense hand movement with a built-in infrared sensor, and some mouse functions are possible via finger motion as if the keyboard is a touchpad mouse [3]. One keyboard can act as a touchpad mouse through touch sensors that

\section{贷 Springer}

(c) 2016 Suzuki et al. This article is distributed under the terms of the Creative Commons Attribution 4.0 International License (http://creativecommons.org/licenses/by/4.0/), which permits unrestricted use, distribution, and reproduction in any medium, provided you give appropriate credit to the original author(s) and the source, provide a link to the Creative Commons license, and indicate if changes were made. 
are placed on the keyboard surface $[4,5]$. These studies considered mouse functions such as cursor movement and scrolling, but studies that examine clicking and dragging functions have not yet been conducted. In order to mount mouse function onto the keyboard, part of the keyboard needs to be made into something like a touchpad mouse. However, mouse function via touchpad has been reported to take more time than that using a conventional mouse [6]. Thus, improving efficiency of typing and mouse operation by integrating the devices, a more useful approach might be to mount the keyboard function onto a mouse that is operated by the hand and fingers.

Several attempts have been made to mount keyboard functions on a mouse. One is a multi-button mouse that can have several keys mounted on it [7]. With this type of mouse, keys such as 'Return' or 'Delete' can be input using buttons on the side of the mouse. CombiMouse, which has the entire right half of a standard keyboard connected to a mouse, can be used similarly as a standard mouse [8, 9]. Character input speed of CombiMouse has been reported to be $70 \%$ that of a standard keyboard. To improve typing and mouse operation efficiency, ideally the keys can be pressed with small finger movements while a hand is placed on the mouse. In contrast, CombiMouse requires the hand to be released from the mouse portion of the device when using the keyboard portion, and a suitable key structure for mounting on a mouse has not yet been considered. DataHand is a mouse equipped with a keyboard with a special concave key structure around the fingertip [10]. Because the concave keys of DataHand can be pressed using small finger motions, the hand can stay on the mouse at all times, even when typing. However, character input speed with DataHand only reached $70 \%$ that of the standard keyboard after practice for more than $13 \mathrm{~h}$. Thus, learning the special key arrangement still presents some difficulties for character input. Based on these attempts of integrating a keyboard onto a mouse, an ideal interface should satisfy the following conditions: (1) the distance that fingers are required to move should be reduced and keys should be accessible while the hand is on the mouse and (2) learning the special key arrangement should be fairly easy. For this purpose, the best key arrangement is one that fits along the fingers and maintains the same direction of finger movement as is employed by the standard keyboard. This should eliminate difficulty learning a special key arrangement. Analyzing finger motion while typing on the standard keyboard is an effective way to determine the best arrangement based on these conditions.

Finger-motion analysis while typing on keyboards has been reported in several studies that measured finger-joint angle, joint-angle velocity, and joint-angle acceleration [11-13]. Although the displacement of finger-joint position trajectory is necessary to determine the key arrangement that requires the least finger movement, these data have rarely been reported.

\section{Objective}

We propose a keyboard-mounted mouse whose keys fit along the fingers, allowing minimal finger movement while the hand is placed on the mouse, and whose key arrangement is easy to learn. The conventional keyboardmounted mouse is operationally satisfactory in terms of mouse function because the mouse is a simple interface that fits the hand nicely and whose only function is the control of cursor movement and selection via mouse click $[8,9]$. However, the keyboard portion of the conventional keyboard-mounted mouse does not fit fingers effectively, and complex operations typically performed with a keyboard are difficult. Thus, slower character-input speed compared with the standard keyboard remains a problem for the conventional keyboard-mounted mouse [8-10]. One solution to this problem might be a keyboard design that fits along fingers, but studies of such a method have not been reported. If keys are to be fitted along fingers, multiple keys must be in close contact with a single finger. Because of this, a method for being able to separately press several different keys with a single finger must be part of the overall design. In this paper, we focus on designing the arrangement of keys that fit along fingers for a keyboard-mounted mouse. Our objective is to design and test a method for separately being able to press several keys with a single finger, and in the process, reduce finger-moving distance. To accomplish this, we tracked fingers while participants typed on a standard keyboard and used the data to derive a proposed key arrangement. We then developed a keyboard structure that incorporated this layout and tested our predictions. We used three-dimensional (3-D) finger-motion analysis to determine the distance that fingers traveled while performing a simple typing task, and recorded typing accuracy (Experiment 1). To determine how accuracy on the proposed keyboard compared with those of a standard keyboard, we computed error rates for participants who performed 20 trials of a typing exercise on each type of keyboard (Experiment 2).

\section{Methods}

\section{Target keys on our keyboard-mounted mouse}

We proposed a keyboard-mounted mouse that is used by the right hand because most people are right-handed and use a mouse with their dominant hands. Character keys are the most frequently used keys on a keyboard. When inputting character keys around the home position of the keyboard, people often "touch type" without actually 
looking at the keys. Normal typing divides the keyboard into a left and right side with the boundary being ' $\mathrm{Y}$ ', ' $\mathrm{H}$ ', and ' $N$ ' on the right and ' $T$, ' $G$ ', and 'B' on the left. However, ' $\mathrm{Y}$ ', ' $\mathrm{H}$ ', and ' $\mathrm{N}$ ' could be typed with the left hand. Therefore, we focused on developing a keyboard for typing 12 characters around the home position on the right

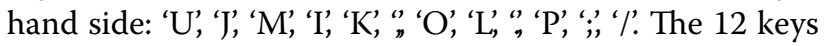
are arranged in the usual four columns, with a different finger assigned to the three keys in each column. Mouse clicks and scrolling are done by assigning the click keys and scroll keys (up and down) to some of the 12 keys. The thumb, which is not used for typing character keys on the standard keyboard, is used for operating the switch that toggles the device's function between keyboard and mouse. The switch not only toggles between keyboard and mouse functions, but can also toggle the key map for the keys, similar to the 'shift' key on a standard keyboard. Thus, the 12 character keys can be made to represent the other keys on the right-hand side of a standard keyboard

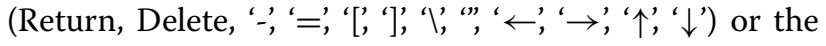
numeric keys (0-9).

\section{Methods for using one finger to press three separate keys}

A 3-D key arrangement that fits along the fingers may be effective for reducing the distance fingers move while typing, but because each finger joint touches different keys at the same time, typing accuracy might be reduced. However, with a careful key-arrangement design that considers the combinations and positions of finger-joint movements, a keyboard can be made such that three keys can be typed separately by each finger joint.

Fingers have three joints, the distal interphalangeal (DIP) joints, proximal interphalangeal (PIP) joints, and metacarpophalangeal (MCP) joints. PIP joints and MCP joints can move voluntarily, and DIP joints move subordinately to PIP joint movement [14]. Therefore, eight voluntary finger motion types are possible by combining two finger joints (MCP and PIP) and two directions of motion (flexion and extension) (Fig. 1a). Observing finger motion on a standard keyboard shows that as a finger moves from the middle key to the upper key, the MCP joint flexes and the PIP joint extends. Touching the middle key requires the MCP joint to flex, and moving to the lower key requires the PIP joint to flex and the MCP joint to extend (Fig. 1b).

Moving some fingers individually is difficult because the finger muscles and corticospinal neurons are linked between fingers [15]. Because finger extension causes more finger-linked movements than flexion [16, 17], using finger extension to separately type keys that are in close contact to fingers is difficult. Therefore, keys on our new keyboard are arranged such that they can be typed using flexion. By considering the finger motions depicted in Fig. 1b, we assumed that 3-D keys placed in the red areas depicted in Fig. 1c could be typed through flexion. We hypothesized that 3-D keys could be typed through flexion with limited finger movement and with as much accuracy as the standard keyboard if the keys in the red areas in Fig. 1c could sense initial finger motion and ignore subsequent interference by other joint movements. By analyzing finger motion, we should be able to determine the appropriate 3-D key positions for sensing the distinct and separate initial finger motions. We did so by determining which joint began moving first and how other joints subsequently moved during normal typing on a standard keyboard. Thus, we reasoned that placing the new keys in positions that sense the first movements of the joints, not interfering with tapping of the other keys, and matching the red areas in Fig. 1c, we would be able to develop a 3-D keyboard that can be used accurately through flexion but that does not require fingers to move as much as a standard keyboard.

\section{Typing motion analysis}

To minimize finger movement and to allow the three keys along each finger to be pressed separately, keys need to be arranged such that they are in close contact with the finger position that starts moving the earliest when typing, and such that extraneous joint movements do not interfere with pressing the target key. Thus, we analyzed typing motion on the standard keyboard. The objective of this experiment was to determine finger positions when typing on a standard keyboard, and then apply them to the 3-D arrangement.

\section{Experimental protocol}

We used the AURORA ${ }^{\circledR}$ magnetic 3-D position measurement system (Northern Digital Inc., Canada), a standard keyboard consistent with the ISO 2530:1975, and palm rest (Fig. 2).

Five healthy men (mean age 21.6 years; range 20-23 years; all right-handed) were asked to input character keys with the index finger, middle finger, ring finger, and little finger of the right hand. Informed consent was obtained from each subject before participation. The 12 characters were typed five times each, with each finger typing three different characters (60 character inputs for each participant). The keys 'J', ' $\mathrm{K}$ ', 'L', and ';' were set as the home position, and participants were asked to return their fingers to these four keys after inputting each character. We measured the 3-D trajectory of fingertips, distal interphalangeal (DIP) joints, proximal interphalangeal (PIP) joints, and metacarpophalangeal (MCP) joints during typing. Task instructions were as follows:away from the other 


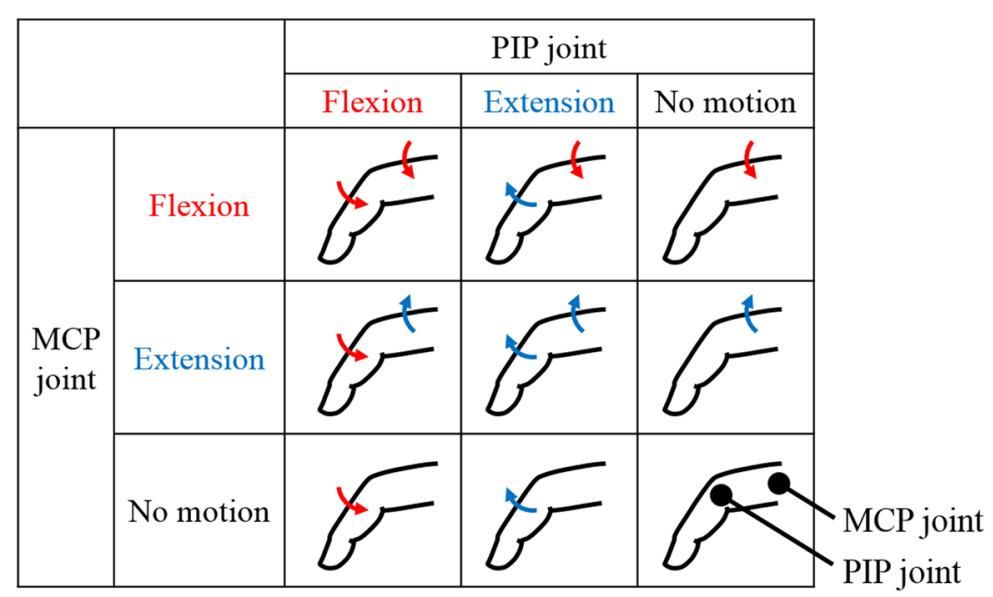

a Eight finger-motion types constructed by combining two finger joints and two directions of motion

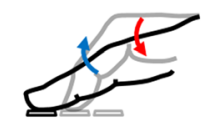

Upper key

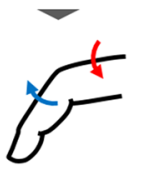

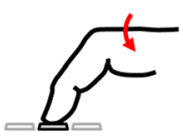

Middle key

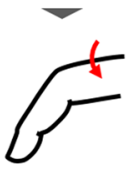

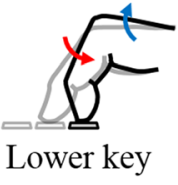

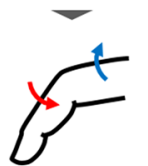

b Finger-motion types for pressing the upper, middle, and lower keys on a standard keyboard

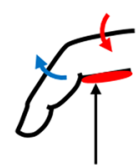

Upper key

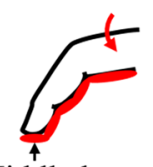

Middle key

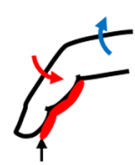

Lower key

c Proposed 3-D key areas for pressing three keys with one finger through flexion

Fig. 1 Proposed 3-D key areas for pressing three keys with one finger through flexion. The findings are based on finger motions constructed by combining two finger joints (MCP and PIP) and two directions of motion (flexion and extension). a Eight finger-motion types constructed by combining two finger joints and two directions of motion, $\mathbf{b}$ finger-motion types for pressing the upper, middle, and lower keys on a standard keyboard, c proposed 3-D key areas for pressing three keys with one finger through flexion

1. Place the palm on the palm rest and place the fingers naturally on the home-position keys.

2. Type the 'U', 'J', and 'M' keys five times each using the index finger.

3. Type the 'I', 'K', and ', keys five times each using the middle finger.

4. Type the 'O', 'L', and '. keys five times each using the ring finger.

5. Type the 'P' and ';', and ' $\%$ ' keys five times each using the little finger.

\section{Results and discussion}

The mean and standard deviation of finger displacement in the $y$ - and z-axes are shown in Fig. 3 (300 character inputs, 60 inputs $\times 5$ subjects). Initial finger position is the origin and has a displacement of zero. When we averaged the data, we normalized the time axis after approximating the data with a linear regression between the two points on the data plot.

Negative displacements in the $y$ - and $z$-axes indicate that the joint was moved by bending another joint. By placing a key at the sites at which joints moved in a 


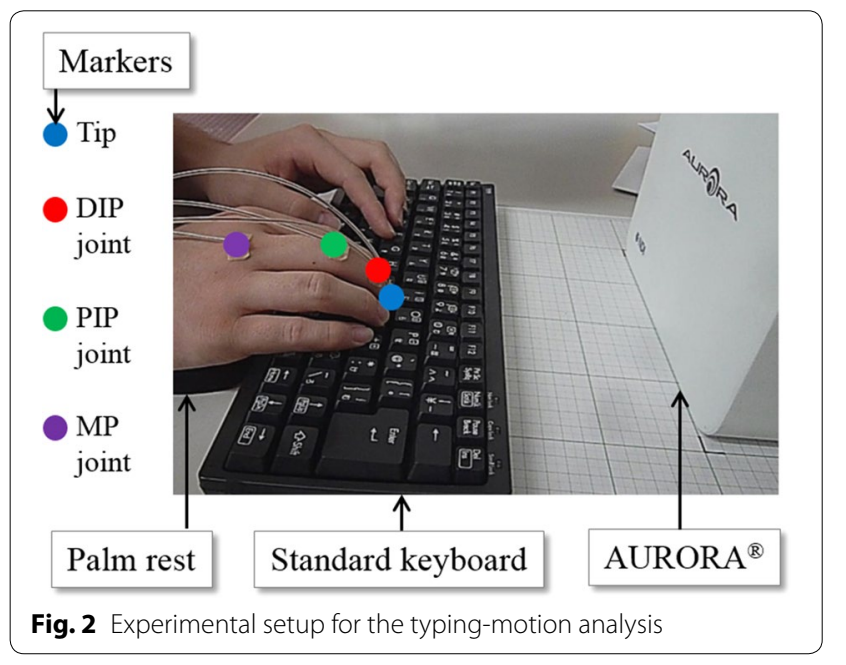

negative direction, the key can be pressed by bending the finger, which produces less finger-linked movements than does extension $[16,17]$. The starting times for such movements are shown in Table 1 and Fig. 4. Because the push-in distance of keys on a standard keyboard is $3.0 \mathrm{~mm}$ [18], the displacement start time was set to the time when the displacement from the initial position reached $3.0 \mathrm{~mm}$. In Table 1, a dash means that there were no negative displacements greater than $3.0 \mathrm{~mm}$ in a given axis.

As seen in Fig. 4a, when typing keys in the upper row, negative displacement in the $\mathrm{z}$-axis is fastest in the PIP joint. The PIP joint starts moving significantly earlier than the DIP and MCP joints ( $\mathrm{df}=4, p=0.001$ vs. DIP joint $\mathrm{z}$-axis, $p=0.02$ vs. MCP joint $\mathrm{z}$-axis). Thus, typing upper-row keys would be sensed earliest by arranging

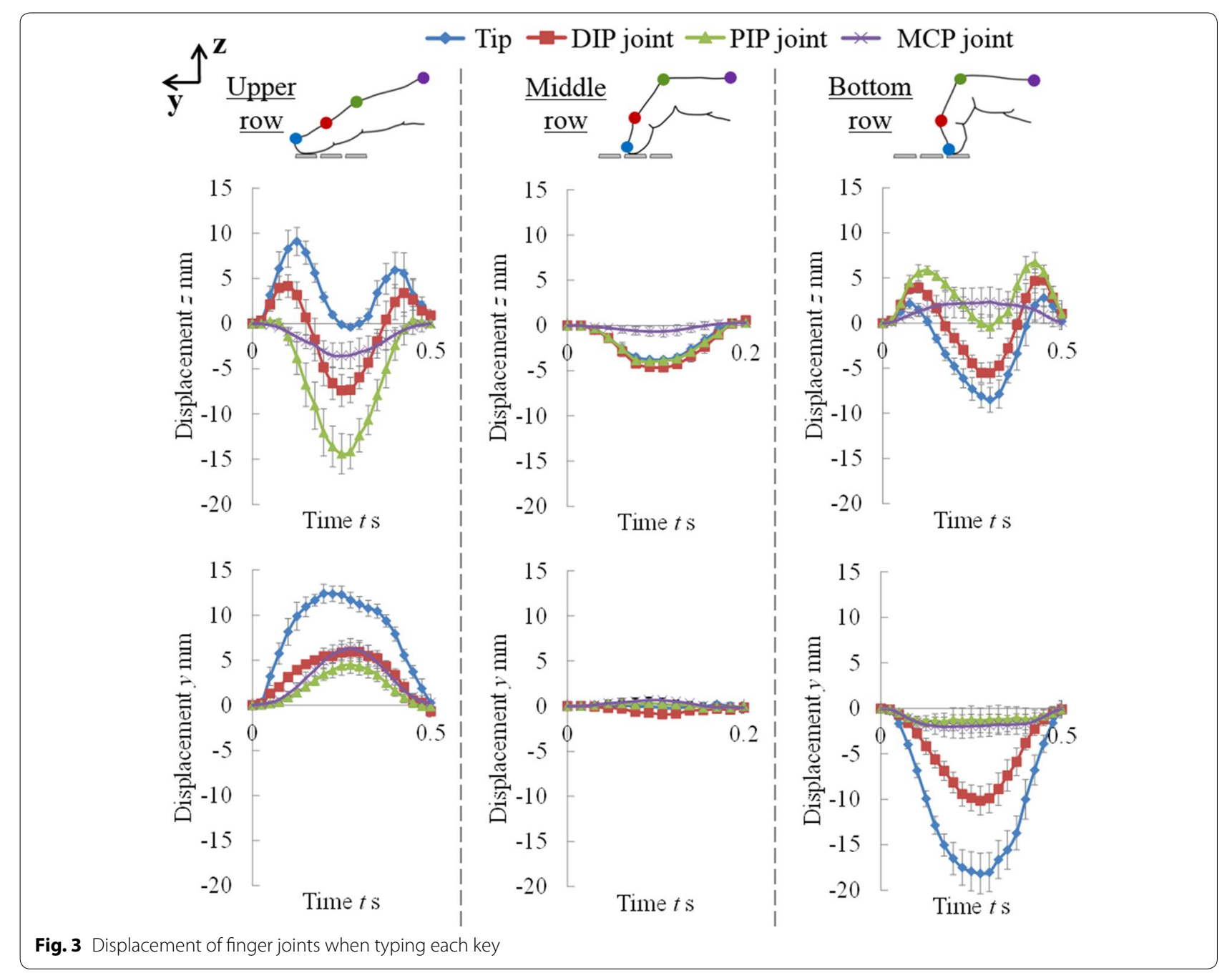


Table 1 Displacement start time of each finger part

\begin{tabular}{lcccc}
\hline & Tip & DIP joint & PIP joint & MCP joint \\
\hline Upper key & & & & \\
y-axis start time (s) & - & - & - & - \\
z-axis start time (s) & - & 0.18 & 0.12 & 0.16 \\
Middle key & & & & \\
y-axis start time (s) & - & - & - & - \\
z-axis start time (s) & 0.068 & 0.062 & 0.067 & - \\
Lower key & & & & - \\
y-axis start time (s) & 0.064 & 0.11 & - & - \\
z-axis start time (s) & 0.17 & 0.22 & - & - \\
\hline
\end{tabular}

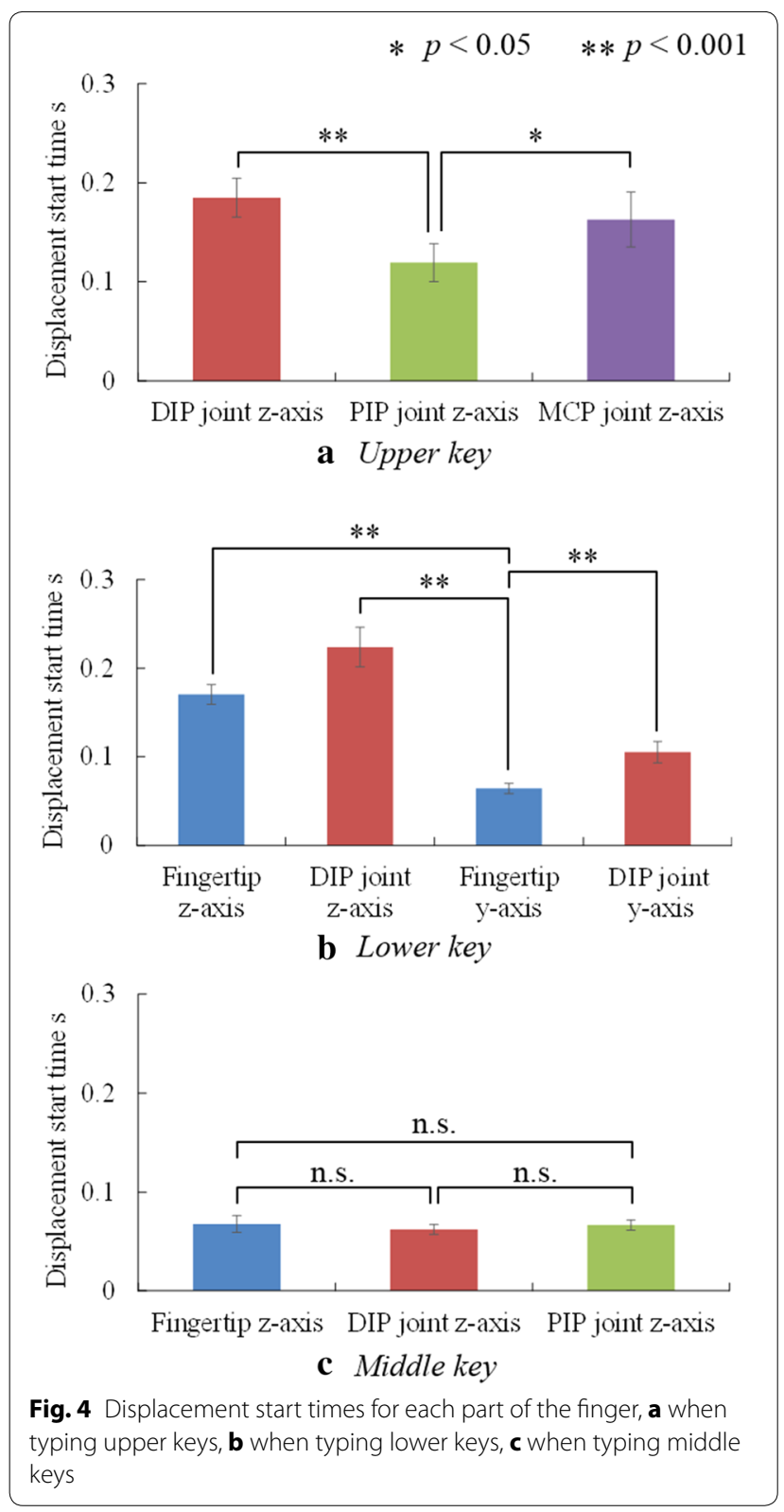

3-D keys in close contact with the PIP joint. Taking into account the red area in Fig. 1c so that the keys can be pressed through flexion, the 3-D keys for upper-row characters should be placed at the proximal phalanx (the part of the finger between the PIP and MCP joints) near the PIP joint on the negative z-axis (Fig. 5, left).

Data from Fig. 4b also show that when typing a character in the bottom (lower) row, negative displacement in the $\mathrm{y}$-axis is significantly early in the fingertip $(\mathrm{df}=8$, $p<0.001$ vs. DIP joint $\mathrm{y}$-axis, $p<0.001$ vs. fingertip $\mathrm{z}$-axis, $p<0.001$ vs. DIP joint $\mathrm{z}$-axis). Taking into account the red area in Fig. 1c so that the keys can be pressed through flexion, the 3-D key for lower-row characters should be placed at the fingertip in the negative $y$-axis (Fig. 5, right).

Figure 4c also indicates that when typing characters from the middle row, onset of negative movement in the $\mathrm{z}$-axis occurs almost at the same time for the fingertip, DIP joint, and PIP joint, and t-tests show no significant differences. Thus, typing middle-row characters can be sensed at the same time by making the 3-D keys in close contact with the fingertip, DIP joint, and PIP joint. Taking into account the red area in Fig. 1c so that the keys can be pressed through flexion, and considering the proposed 3-D key placements for upper and lower keys, the middle 3-D key should be placed either in the same position as on a standard keyboard or at the middle phalanx (the part of the finger between the DIP and PIP joints) (Fig. 5, center).

We next used the data to verify that the proposed 3-D key positions can be pressed separately with a single finger. PIP joint movement used to press an upper key cannot interfere with other proposed keys because when the PIP joint moves to press an upper key, the fingertip moves in the positive direction in the $y$ - and $z$-axes (Fig. 3), which is away from the other proposed keys. Fingertip movement to press a lower key might interfere with a middle key placed at the middle phalanx because when the fingertip moves to press the lower key, the DIP joint moves in the negative direction in the y-axis (Fig. 3). In contrast, fingertip movement to press a lower key cannot interfere with other keys if the middle key is placed in the same position as on a standard keyboard because fingertip movement is in a positive direction in the $\mathrm{z}$-axis, which avoids pressing a middle key, and the PIP joint moves in a positive direction in the $\mathrm{z}$-axis, which avoids pressing the upper key (Fig. 3).

Fingertip movement to press a middle key placed in the same position as on a standard keyboard or at the middle phalanx might interfere with other keys because the PIP joint and fingertip move in the negative direction in the z-axis (Fig. 3). However, because pressing a middle key requires only a small movement, by modifying the 


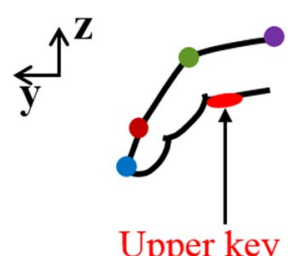

Fig. 5 Proposed areas for placing the keys

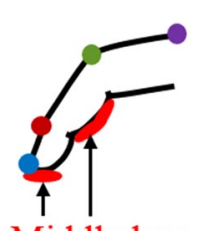

Middle key

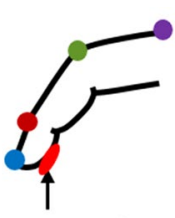

Lower key other key positions a little so that they cannot sense when the fingertip presses the middle key, fingertip movement should not interfere with the other keys.

In conclusion, the 3-D key arrangement, shown in Fig. 6 can sense the initial typing motion and pressing a target key does not cause other keys to be pressed, provided that upper and lower keys are set so that they do not sense the force applied when the user presses a middle key.

Upper key: Vertically below the PIP joint of the proximal phalanx.

Middle key: Vertically below the fingertip (in the same position as on a standard keyboard).

Lower key: At the fingertip when the PIP joint is flexed.

\section{3-D keyboard design}

\section{Hardware}

According to the typing-motion analysis, we designed a 3-D keyboard structure as depicted in Fig. 7, and developed it as shown in Fig. 8. A Flexi Force Sensor ${ }^{\circledR}$ (Tekscan Inc., USA) was built into each 3-D key, which could then sense the force of each finger. An Arduino Leonardo board (Arduino Srl, Italy) was used as a microcomputer to handle the A/D conversion of the force signal. The 3-D keys have adjustable mechanisms that allow the position and angle to be custom fit to a user's fingers. The optimal positions put the keys in close contact with the fingers so that the initial typing motion is sensed quickly. The upper and lower keys are set to a position such that the upperand lower-key output from the force sensors become $0 \mathrm{~N}$ when the user presses the middle key. The key pitch of the 3-D keyboard was set to $19 \mathrm{~mm}$, which is the pitch of most keyboards. To reduce the distance fingers move, input is accomplished solely by touching, and pressing is not necessary. The 3-D keyboard corresponds to the character keys that would normally be pressed by the right hand on a standard keyboard (Fig. 9). Thus, the 3-D keyboard is used in conjunction with a standard keyboard. Figure 10 shows an overview of the proposed system.

\section{Character input algorithm}

The force sensor built into the 3-D keys senses the contact force applied by the fingers. When this force exceeds the threshold force, the character code of the key is sent to the computer. The flow chart of the character input algorithm is shown in Fig. 11.

Moving some fingers individually is difficult because some finger muscles and corticospinal neurons are linked between fingers, and the amount of finger linkage can vary from person to person [15]. Because the 3-D keys are in close contact with the fingers, the user may unintentionally press undesired keys because intentional movements of one finger are accompanied by unintentional movements of linked fingers. To prevent erroneous inputs resulting from finger linkage, the force threshold needs to be set individually for each person, depending on how much finger-linkage movement they experience. We therefore devised the following calibration method that can be used to set the threshold force for each individual before initial use:

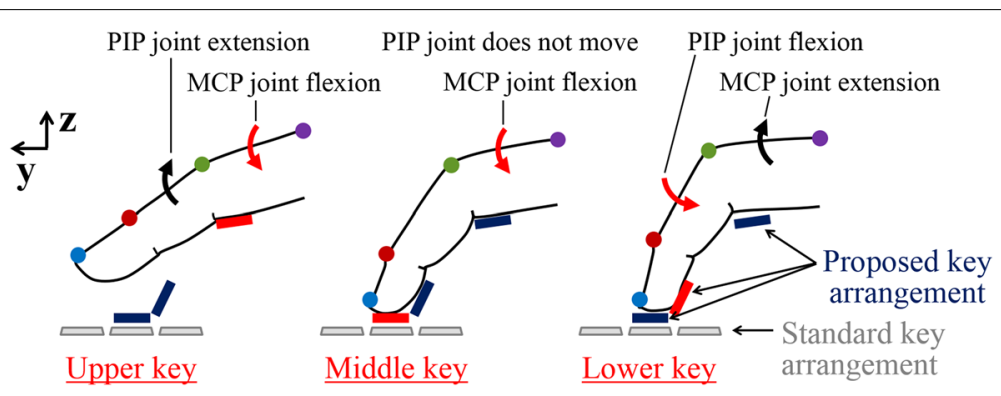

Fig. 6 Proposed key layout and finger motions used to press the keys 

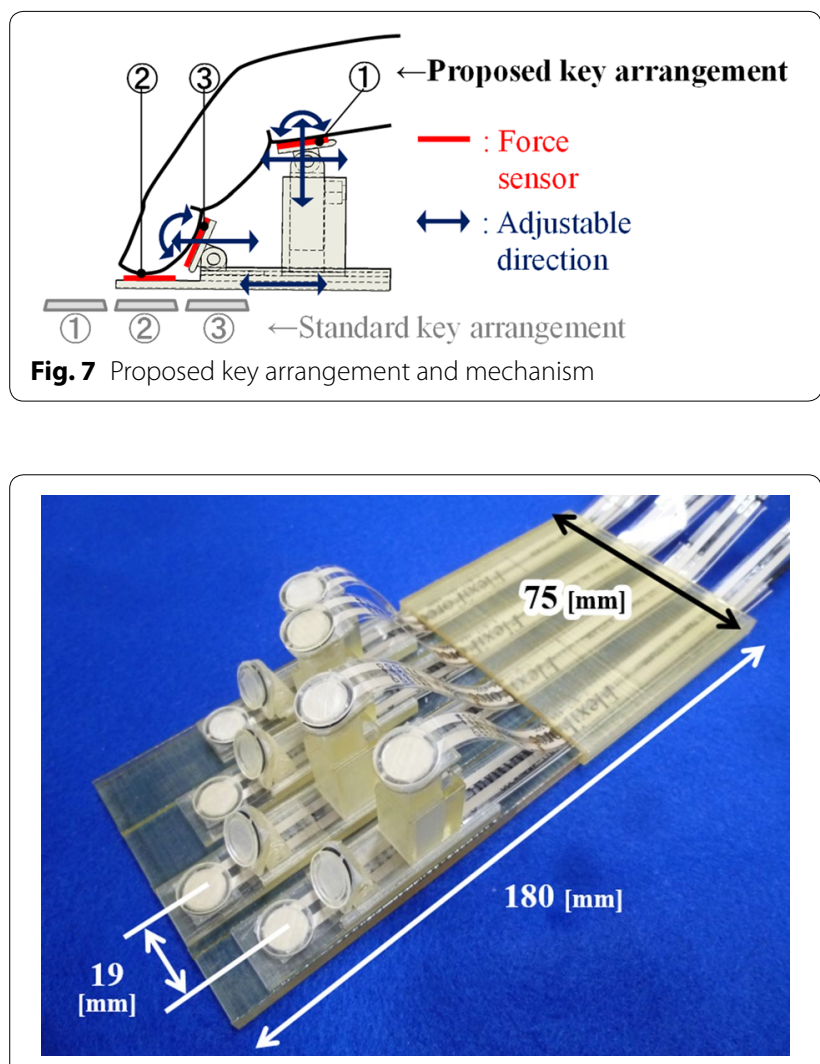

Fig. 8 Three-dimensional keyboard

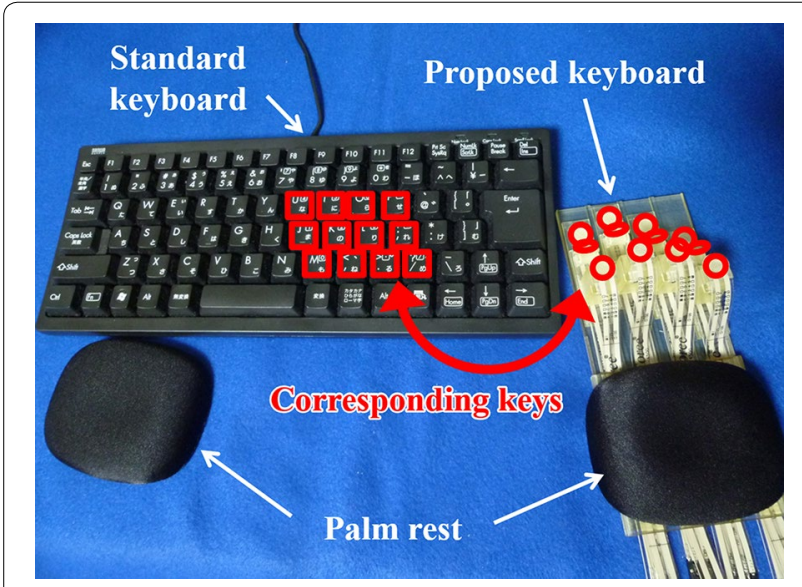

Fig. 9 Placement of proposed keyboard

1. Users place their hand on the keys.

2. Each key position is adjusted so as to be in close contact with the fingers.

3. Users press each 3-D key five times, and the output of the force sensor is recorded (Fig. 12).

4. The maximum contact force $\mathrm{F}_{\max } \mathrm{N}$ that was generated by unintentional touches is calculated.
5. Threshold is set to $\mathrm{F}_{\max }+0.1 \mathrm{~N}(0.1 \mathrm{~N}$ is the force sensor specification for the maximum value of repeatability).

With correct calibration, only character codes from keys that are touched intentionally will be sent to the computer.

\section{Experiment 1: Evaluation of the distance moved while typing and typing accuracy \\ Experimental objective}

The 3-D keyboard is designed to reduce the distance fingers must travel while typing, without reducing typing accuracy caused by inadvertently pressing undesired keys. The objective of this experiment was to evaluate whether the distance that fingers move when using the 3-D keyboard is less than that when using a standard keyboard, and whether the 3-D keyboard can be used as accurately. This was accomplished by measuring 3-D finger motion and the registered input when typing in a regular order.

\section{Experimental protocol}

We used the same equipment as was used in the analysis of typing motion. Participants were three healthy men (mean age 22.3 years; all right-handed). The experiment began after adjusting the keys to be in close contact with the fingers and performing the threshold calibration described above. The task was almost the same as in the typing-motion analysis experiment, except that each key was hit ten times. We measured the 3-D trajectory of the fingertips, DIP joints, PIP joints, and MCP joints during typing. The input characters were recorded in a text file. This experiment was carried out after obtaining informed consent from the subjects.

\section{Results}

The comparison of distance moved while typing each character key (upper, middle, and lower rows) is shown in Fig. 13. The distance for one character input was calculated from the 3-D position as the total distance moved by each finger joint in one finger when touching one key. Means and standard deviations were calculated from the ten trials for each key.

T-tests showed that when using the 3-D keyboard, the distance fingers traveled was reduced significantly in all keys compared with the standard keyboard $(\mathrm{df}=4$, $p=0.03$ for upper-row keys, $p=0.007$ for middle-row keys, $p=0.02$ for lower-row keys). On average, distance was reduced $76 \%$ for the upper-row keys, $58 \%$ for the middle-row keys, and $88 \%$ for the lower-row keys. When all keys were combined, the average reduction was 


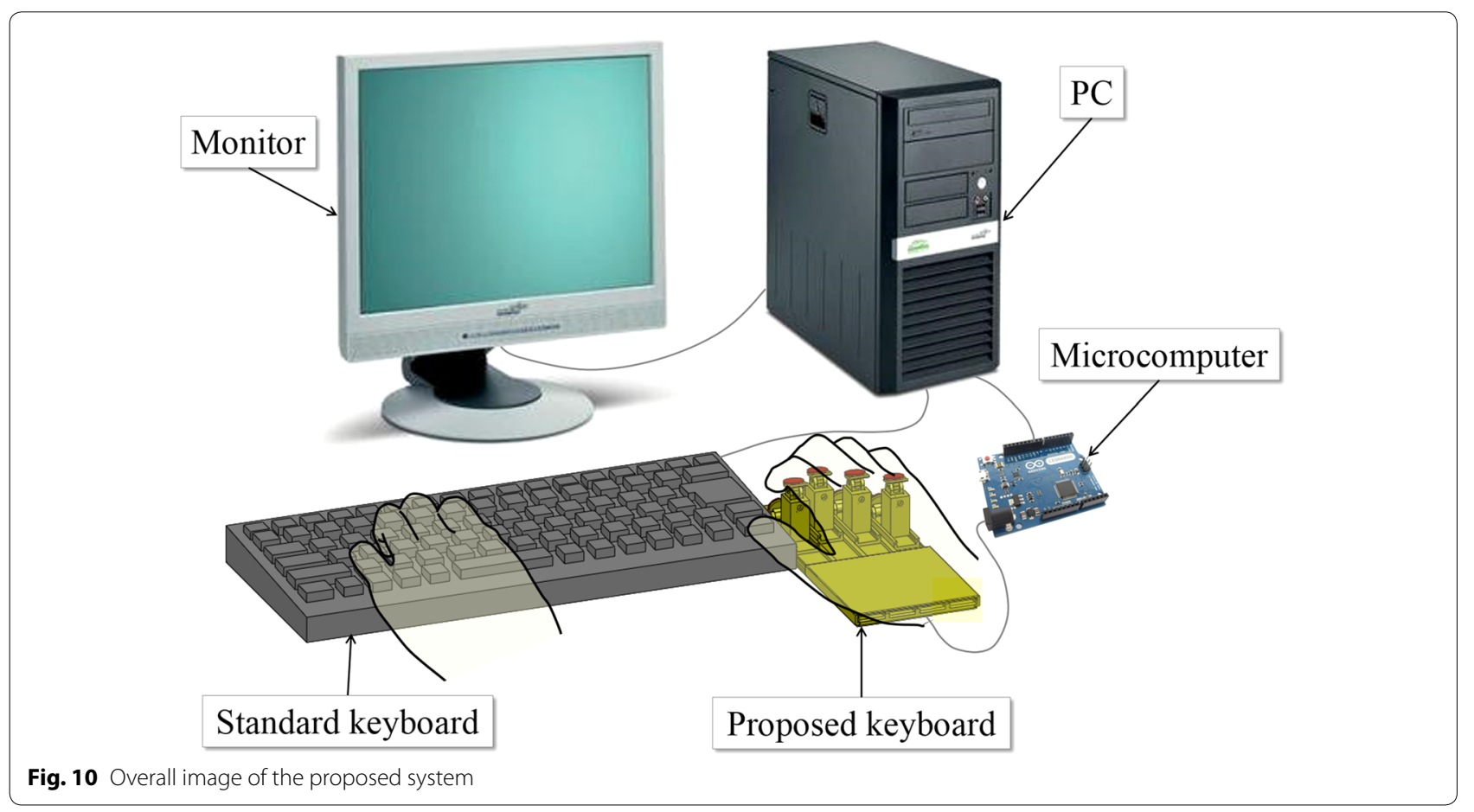

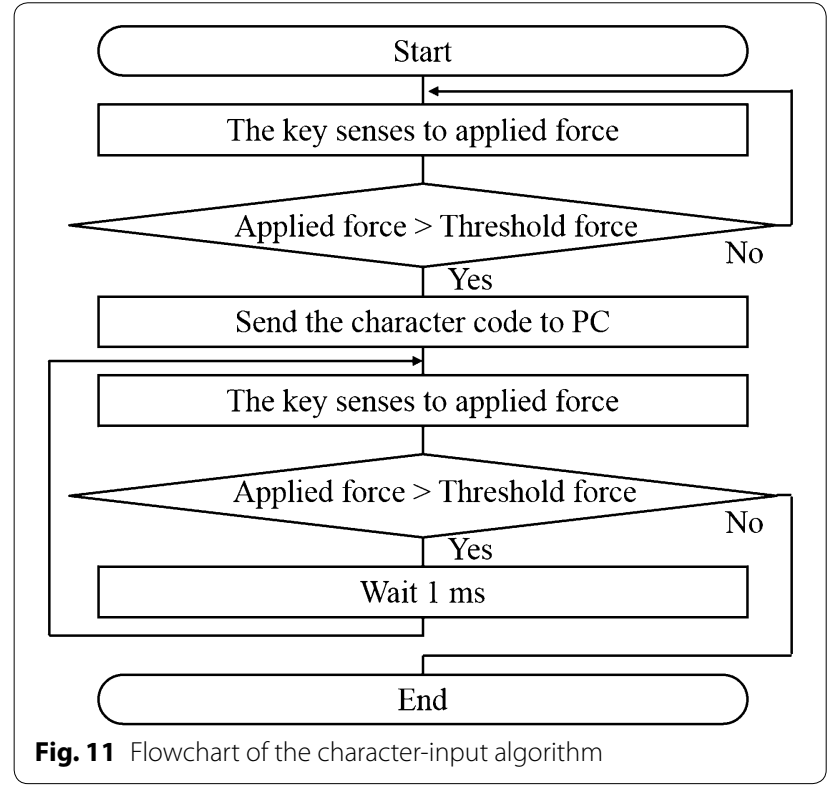

$74 \%$. Participants did not make any typing errors in this experiment.

\section{Experiment 2: Evaluation of typing accuracy when typing a series of characters \\ Experimental objective}

In Experiment 1, we found that typing with 3-D keys is accurate when typing in a regular order. However, this is very simple and differs from actual keyboard use. In Experiment 2, our objective was to compare typing accuracy between the 3-D and standard keyboards when typing a series of characters.

\section{Experimental protocol}

We used a 3-D keyboard and a standard keyboard in conjunction with a Windows XP computer running the software "Stamina Typing Tutor 2.5 [19]", which can record error rate and typing speed.

Five healthy individuals (four men, one woman; mean age 29.2 years; range 20-54 years; four righthanded, one left-handed) who use standard keyboards daily participated in the experiment. None had experience using a 3-D keyboard. The experiment began after adjusting the key positions so that they were in close contact with the fingers and after performing the force-threshold calibration. Thirty pseudorandomly chosen characters were displayed on the monitor, and the task was to type them as fast as possible. To make trial difficulty consistent, the characters alternated between keys on the left and right sides of the keyboard (Fig. 14). Participants completed 40 sets, alternating between the standard and 3-D keyboards (20 sets each). A $15 \mathrm{~s}$ break was taken between each set. Each subject used the 3-D keyboard for about $20 \mathrm{~min}$. The experiment was approved by Waseda University IRB (\#2014-018) and was carried out after obtaining informed consent from the subjects. 

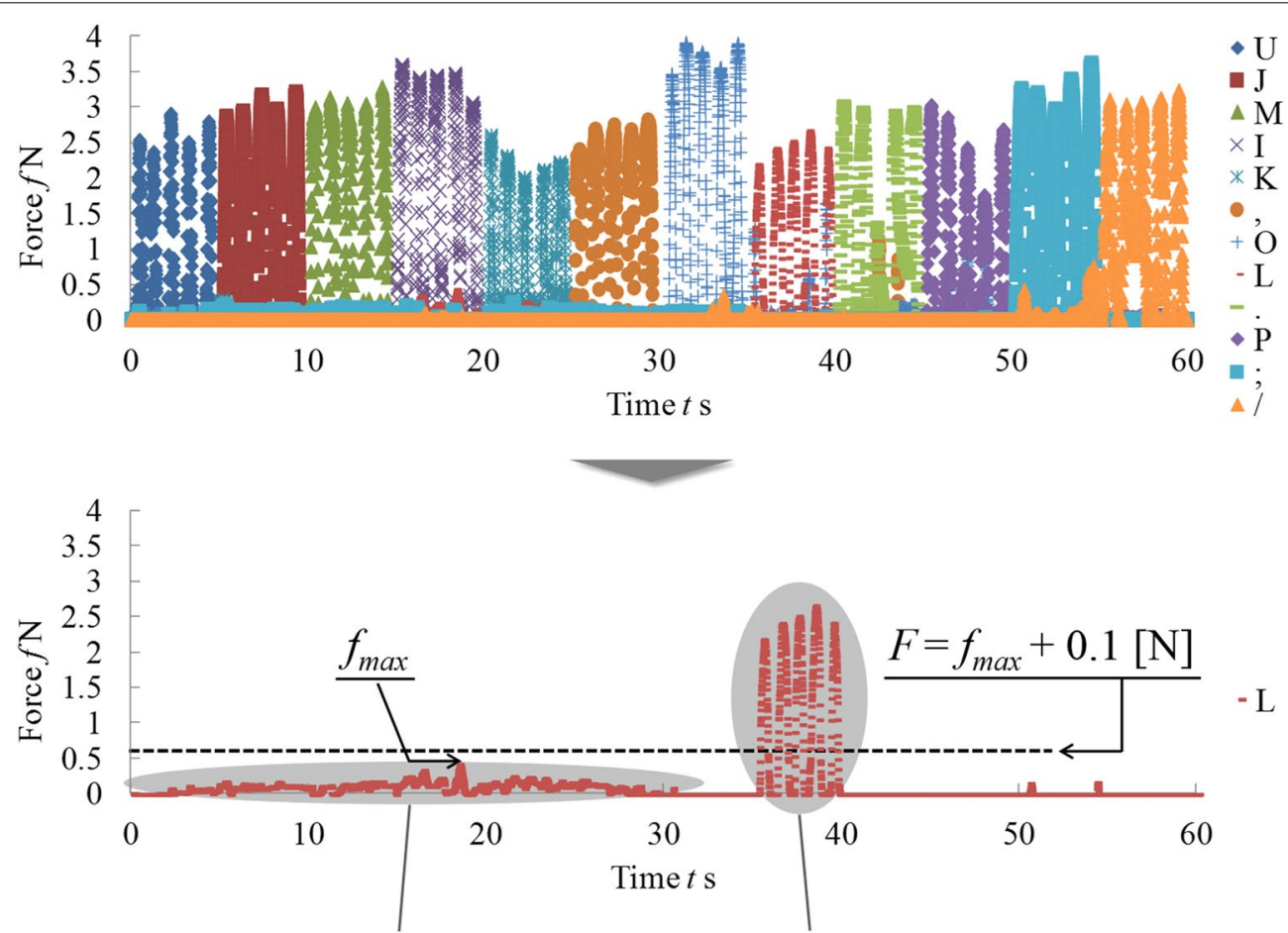

\section{Unintentional contact force caused by finger linkage}

\section{Intentional contact force for inputting characters}

Fig. 12 Force applied to the 'L'key

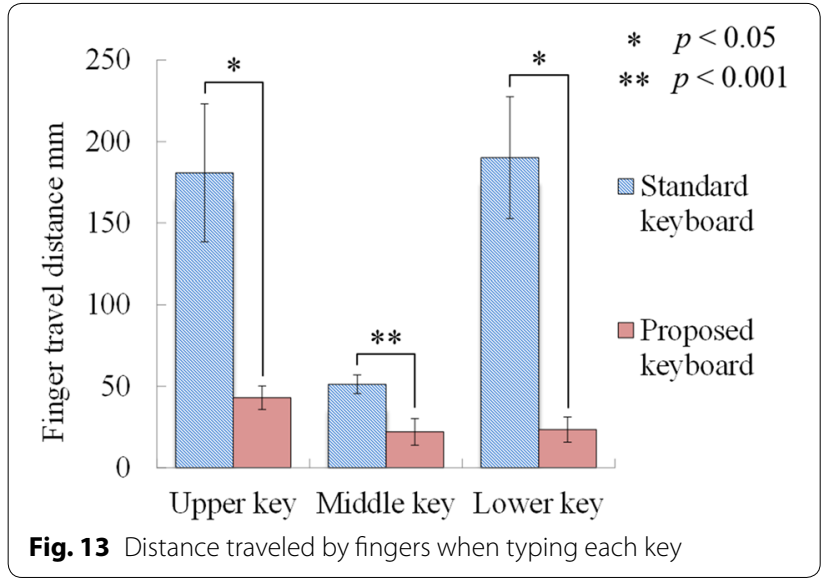

\section{Results}

The total average error rate using the standard keyboard and error rates for each trial on the 3-D keyboard are shown in Fig. 15 for each participant. Comparing the 1st and 20th trial on the 3-D keyboard with t-tests showed that after about $20 \mathrm{~min}$ of practice, the error rate significantly decreased $(\mathrm{df}=8 ; p=0.004)$. The average error rate for the final trial when using the 3-D keyboard was $18 \%$, while that for the first trial was $49 \%$. In fact, subject

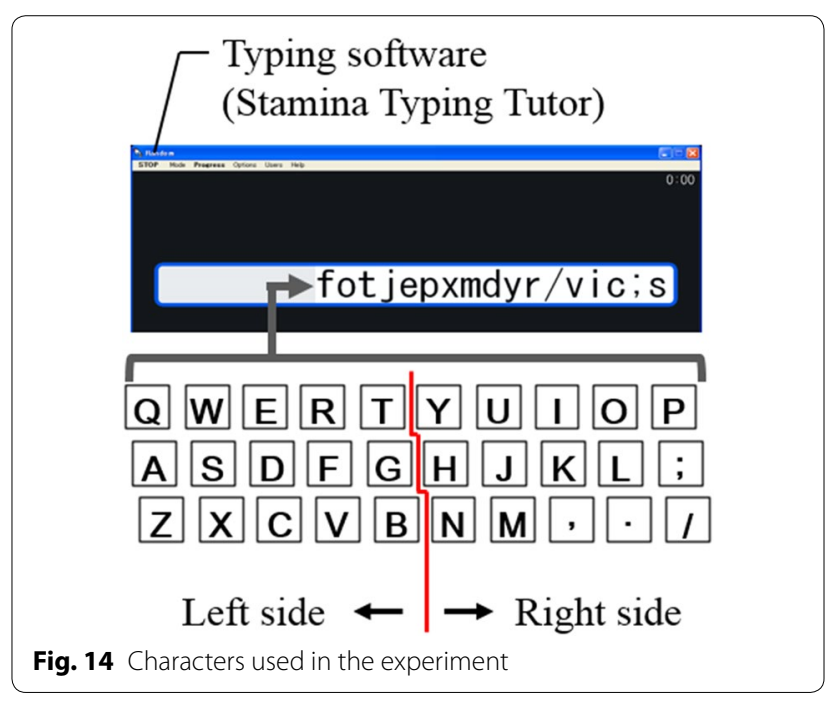

B did not make any mistakes in the 10th trial and subject $\mathrm{C}$ had perfect performance in the 13th. The average error rate across all trials for the standard keyboard was $9.5 \%$.

Typing speed for each subject was calculated and is shown in Fig. 16. Comparing the 1st and 20th trial, typing speed significantly increased $(\mathrm{df}=8 ; p=0.002)$. By 
$\rightarrow$ Subject $\mathrm{A} \rightarrow$ Subject $\mathrm{B} \rightarrow$ Subject $\mathrm{C}$

$*$ Subject D $\rightarrow$ Subject E --- Average for the standard keyboard

O Average for the first trials on the proposed keyboard

- Average for the last trials on the proposed keyboard

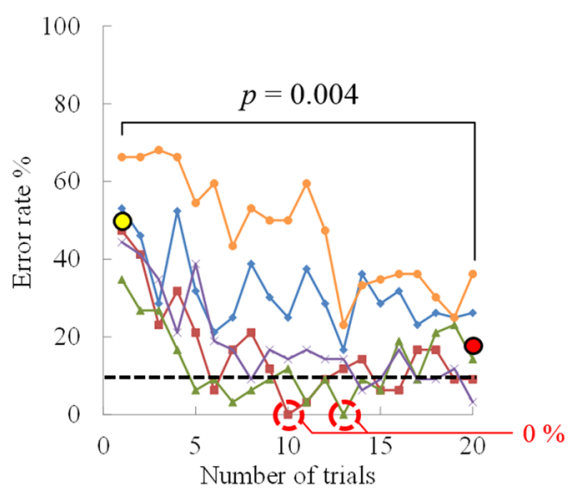

Fig. 15 Error rate across 20 trials

$\rightarrow$ Subject $\mathrm{A} \rightarrow$ Subject $\mathrm{B} \rightarrow$ Subject $\mathrm{C}$

$*$ Subject D $\rightarrow$ Subject E --- Average for the standard keyboard

O Average for the first trials on the proposed keyboard

- Average for the last trials on the proposed keyboard

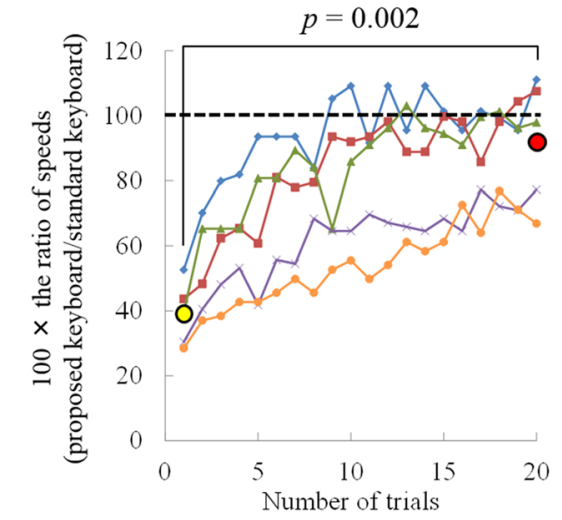

Fig. 16 Typing speed across 20 trials

the 20th trial, the average typing speed when using the 3 -D keyboard reached $92 \%$ of the speed when using the standard keyboard, an increase from $39 \%$ observed at the first trial. For participants A, B, and C, the maximum typing speed with the 3-D keyboard was slightly higher than the average speed when using the standard keyboard.

\section{Discussion}

In Experiment 1, we found that our 3-D keyboard can be used accurately, and that joint movement does not interfere with pressing the target key. Thus, our proposed method satisfies the condition that three keys can be pressed separately by a single finger despite close contact with all three keys.
In Experiment 2, two subjects were able to type 30 characters without any mistakes once in the 20 trials. This result shows that the 3-D keyboard can be used accurately even when typing a series of characters. While the initial trials contained more errors because subjects did not yet recognize the arrangement of keys, this type of mistake decreased as subjects got used to layout. By the last trial, the average error rate was only $18 \%$. Thus, 20 min of using the proposed keyboard reduced the error rate from 49 to $18 \%$.

This error rate was almost double that for the standard keyboard. This can be attributed to a software error in the proposed keyboard. Some erroneous inputs were generated by contact forces that exceeded the input threshold during the experiment. These errors seem to have been caused by finger-linkage motion that was different from that when calibrating the force threshold. The degree of finger linkage might have changed because finger movements changed as subjects became habituated to the 3-D keyboard. In the future, we should devise a method for updating the input-force threshold according to any adaptations to the 3-D keyboard made by the participants. Because the erroneous inputs were caused by finger linkage, further consideration will have to be given to pattern recognition methods that use a combination of force sensors to differentiate intentional input from unintentional input.

In Experiment 2, we also calculated typing speed. Average typing speed during the last trial on the proposed keyboard reached $92 \%$ of that on the standard keyboard. Some participants reached maximum typing speeds on the 3-D keyboard that were faster than the average typing speed on the standard keyboard. Therefore, about $20 \mathrm{~min}$ of practice is all that is required to become accustomed to the 3-D keyboard and reach a typing speed equivalent to that of a standard keyboard. Changes in the software could improve the final speed and rate of improvement within the same amount of practice time.

Although we found some significant differences, the sample size was quite small. Therefore, we should conduct further experiments with larger sample sizes.

Here, we only focused on the keyboard portion of the keyboard-mounted mouse. In the future, we must equip the mouse function on the 3-D keyboard, and we must make the mechanism and algorism for switching the function between keyboard and mouse, or between different sets of keys. We also need experiments that evaluate the operability of our new device in tasks using both the keyboard and mouse.

\section{Conclusion}

Our objective was to design and test a 3-D keyboard design that allows each finger to control three separate 
keys, and in the process, reduce finger-moving distance. We analyzed 3-D finger motion while subjects typed on a standard keyboard, and used these data to develop a keyboard that accomplishes this goal. According to the results, the 3-D keys can be pressed separately without interference from other joint movements. Additionally, the distance that fingers moved when using the proposed keyboard was $74 \%$ less than that when using the standard keyboard. Moreover, after using the proposed keyboard for about $20 \mathrm{~min}$, the average input error rate was reduced to $18 \%$. We conclude that our proposed 3-D keyboard reduces the distance fingers move while typing and allows individual fingers to control three separate keys. In the future, we will devise a better character input algorithm that eliminates erroneous input caused by finger linkage. Further, we must add mouse functionality and the switching mechanism that toggles between keyboard and mouse functions. Then we will evaluate the operability of our proposed keyboard-mounted mouse when using both keyboard and mouse functions.

\section{Authors' contributions}

TS conceived the study, developed the device, carried out all experiments, analyzed the data, and drafted the manuscript. SM, YK and MF participated in the research design and sequence alignment. All authors read and approved the final manuscript.

\section{Author details}

${ }^{1}$ The Graduate School of Science and Engineering, Waseda University, Tokyo, Japan. ${ }^{2}$ The Faculty of Science and Engineering, Waseda University, Tokyo, Japan.

\section{Acknowledgements}

This research was supported in part by the Outstanding Graduate COE Support Subsidy "Global Robot Academia (GRA)", a Grant-in-Aid for Scientific Research (A) (26242061), and a Grant-in-Aid for Scientific Research (S) (25220005). All grants were issued by the Ministry of Education, Culture, Sports, Science and Technology in Japan.

\section{Competing interests}

The authors declare that they have no competing interests.

Received: 23 January 2015 Accepted: 11 December 2015

Published online: 19 January 2016

\section{References}

1. Atkinson S, Woods V, Haslam RA, Buckle P (2004) Using non-keyboard input devices: interviews with users in the workplace. Intern J Ind Erg 33:571-579

2. Armbrüster C, Sutter C, Ziefle M (2007) Notebook input devices put to the age test: the usability of trackpoint and touchpad for middleaged adults. Ergonomics 50(3):426-445

3. Keskin C, Hilliges O, Izadi S, Helmes J (2014) Type-Hover-Swipe in 96 bytes: a motion sensing mechanical keyboard. ACM CHI conference on human factors in computing systems, p 1695-1704
4. Tung YC, Cheng TY, Yu NH, Chen MY (2014) FlickBoard: enabling trackpad interaction with automatic mode switching on a capacitive-sensing keyboard. UIST'14 Adjunct proceedings of the adjunct publication of the 27th annual ACM symposium on user interface software and technology, p 107-108

5. Habib I, Berggren N, Rehn E, Josefsson G, Kunz A, Fjeld M (2009) DGTS: integrated typing and pointing. Human-computer interaction-INTERACT, Volume 5727 of the series Lecture Notes in Computer Science, $p$ 232-235

6. Ulrich TA, Boring RL, Lew R (2015) Control board digital interface input devices - touchscreen, trackpad, or mouse. Resilience Week (RWS), p 1-6

7. Scarlett D (2005) Ergonomic mice: comparison of performance and perceived exertion. Usability news from Software Usability Research Laboratory at Wichita State University

8. Slocum J, Thompson S, Chaparro B, Bohan M (2013) Evaluating the CombiMouse: a new input device for personal computers. Usability news from Software Usability Research Laboratory at Wichita State University

9. Slocum J, Thompson S, Chaparro B, Bohan M (2004) Examining first-time usage of the CombiMouse ${ }^{\mathrm{TM}}$. In: Proceedings of the human factors and ergonomics society 48th annual meeting

10. Knight LW, Retter D (1989) DATAHAND: design, potential performance, and improvements in the computer keyboard and mouse. In: Proceedings of the human factors society 33rd annual meeting, p 450-454

11. Baker Nancy A, Cham Rakie, Hale Erin, Cook James, Redfern Mark S (2007) Digit kinematics during typing with standard and ergonomic keyboard configurations. Intern J Ind Erg 37:345-355

12. Baker Nancy A, Cham Rakie, Cidboy Erin Hale, Cook James, Redfern Mark $S$ (2007) Kinematics of the fingers and hands during computer keyboard use. Clin Biomech 22:34-43

13. Soechting John F, Flanders Martha (1997) Flexibility and repeatability of finger movements during typing-analysis of multiple degrees of freedom. J Comput Neurosci 4:29-46

14. Leijnse JNAL, Quesada PM, Spoor CW (2010) Kinematic evaluation of the finger's interphalangeal joints coupling mechanism — variability, flexionextension differences, triggers, locking swanneck deformities, anthropometric correlations. J Biomech 43:2381-2393

15. Aoki Tomoko, Francis Peter R, Kinoshita Hiroshi (2003) Differences in the abilities of individual fingers during the performance of fast repetitive tapping movements. Exp Brain Res 152(2):270-280

16. Yu WS, van Duinen H, Gandevia SC (2010) Limits to the control of the human thumb and fingers in flexion and extension. J Neurophysiol 103:278-289

17. Schieber MH (1991) Individuated finger movements of rhesus monkeys: a means of quantifying the independence of the digits. J Neurophysiol 65(6):1381-1391

18. Kim JH, Aulck L, Bartha MC, Harper CA, Johnson PW (2014) Differences in typing forces, muscle activity, comfort, and typing performance among virtual, notebook, and desktop keyboards. Appl Ergon 45(6):1-8

19. Stamina typing tutor by typingsoft. http://typingsoft.com/stamina.htm. Accessed 10 Jan 2015

\section{Submit your manuscript to a SpringerOpen ${ }^{\circ}$ journal and benefit from:}

- Convenient online submission

- Rigorous peer review

- Immediate publication on acceptance

- Open access: articles freely available online

- High visibility within the field

- Retaining the copyright to your article

Submit your next manuscript at $\downarrow$ springeropen.com 